PHYSICAL REVIEW D 96, 129903(E) (2017)

\title{
Erratum: Cauchy-horizon singularity inside perturbed Kerr black holes [Phys. Rev. D 93, 041501(R) (2016)]
}

Lior M. Burko, Gaurav Khanna, and Anıl Zenginoğlu

(Received 1 December 2017; published 26 December 2017)

DOI: 10.1103/PhysRevD.96.129903

In this Erratum we correct the behavior of the Weyl scalar $\psi_{4}$ and of the linearized curvature scalar $K:=R_{\alpha \beta \gamma \delta} R^{\alpha \beta \gamma \delta}$ approaching the mass-inflation singularity along an outgoing null direction. In the original paper we used a second-order code, that allowed us to find very accurately the fields in the exterior and on the event horizon. However, it has some disadvantages approaching the Cauchy horizon $(\mathrm{CH})$. Specifically, our code solves for $\Delta^{-2} \psi_{4}$, which diverges exponentially with advanced time approaching the $\mathrm{CH}$ (more specifically, diverges like $e^{2 v / M}$ ). Moreover, in the code's coordinates this field
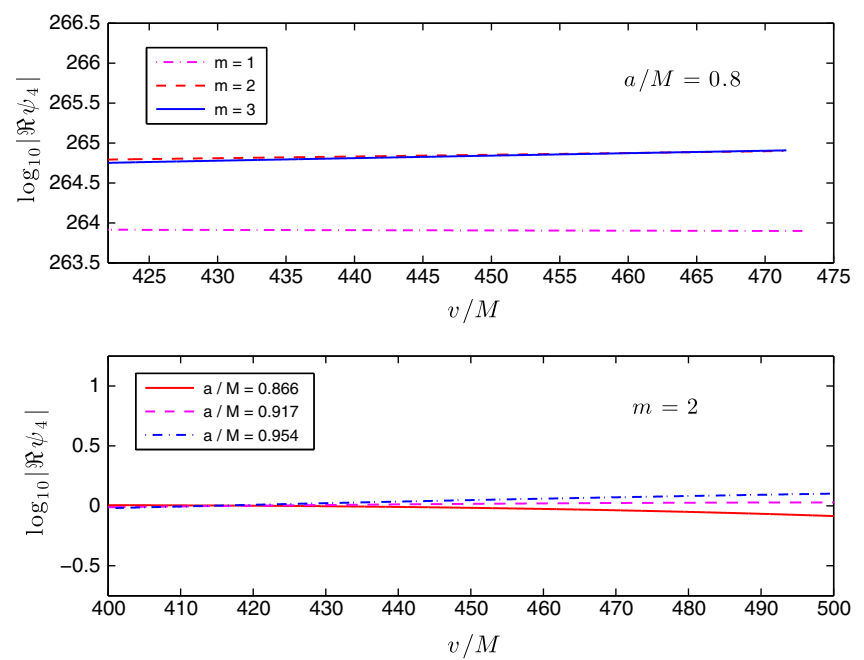

FIG. 7. The magnitude of (the real part of) $\psi_{4}$ in ingoing Kerr coordinates as a function of advanced time along an outgoing null ray. Top panel: The cases $m=1$ (dash-dotted curve), $m=2$ (dashed curve), and $m=3$ (solid curve) for $a / M=0.8$. Bottom panel: The cases $a / M=0.866$ (solid curve), $a / M=0.917$ (dashed curve), and $a / M=0.954$ (dash-dotted curve) for $m=2$, in normalized units. Note, that here we show only the magnitude of $\psi_{4}$, factoring out its oscillations.
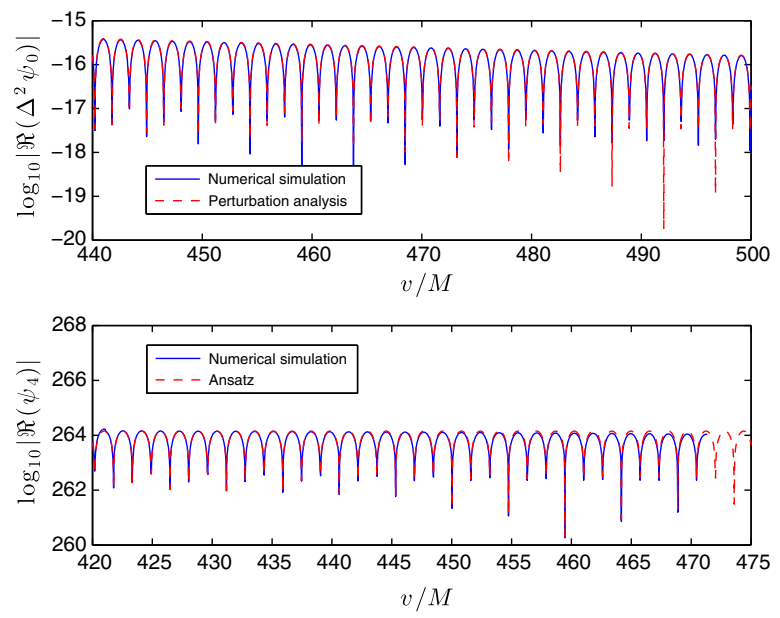

FIG. 5. The Weyl scalars $\Delta^{2} \psi_{0}$ in regularized coordinates (upper panel) and $\psi_{4}$ in ingoing Kerr coordinates (lower panel) as functions of $v / M$ along an outgoing null ray that intersects with the early part of the Cauchy horizon (as $v / M \rightarrow \infty)$. For $\Delta^{2} \psi_{0}\left(\psi_{4}\right)$ we present in addition to our numerical results in a solid curve also the prediction of perturbation analysis (corrected Ansatz) in a dashed curve. Here, $a / M=0.8$ and $m=2$ and the fields are on the equatorial plane. 


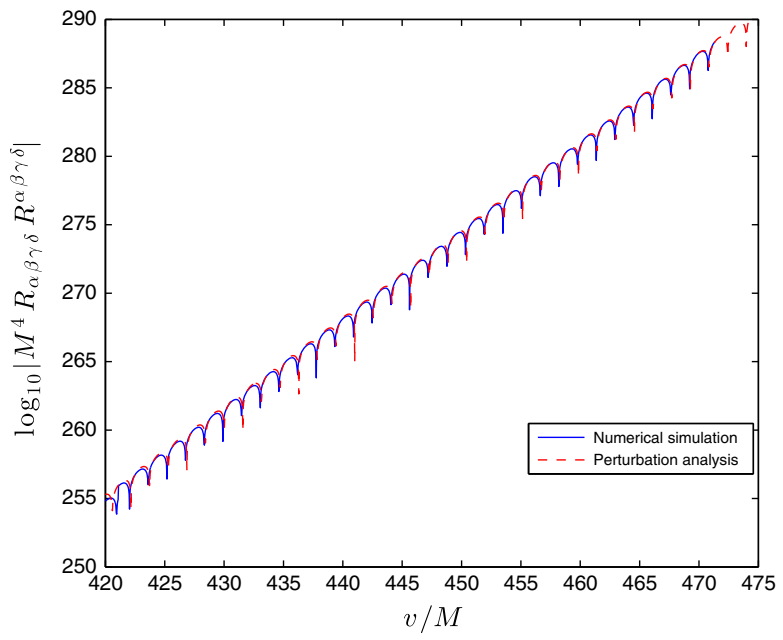

FIG. 6. The curvature scalar $R_{\alpha \beta \gamma \delta} R^{\alpha \beta \gamma \delta}$ as a function of $v / M$ along an outgoing null ray on the equatorial plane approaching the Cauchy horizon. Here, $a / M=0.8$ and $m=2$, and data are on the equatorial plane. The numerical simulation is shown with a solid curve, and the prediction of [6] with a dashed curve.

oscillates approaching the $\mathrm{CH}$. To increase accuracy, we developed a new fifth-order weighted essentially non-oscillatory (WENO) finite-difference scheme [1] with third-order Shu-Osher explicit time-stepping [2,3], which we also used in [4]. More importantly, we improved the treatment of the inner boundary of the computational domain, which is the outgoing leg of the inner horizon (OIH) (see Fig. 1). Specifically, the fields are now actually "evolved" on the inner boundary as opposed to computed using the boundary conditions in conjunction with data from the "bulk." In the original paper we used the latter approach. As we expect sharp physical features to be present at the boundary [5], the approach used here allows for more accurate numerical solutions. In practice, the source-side of the Teukolsky equation is computed at the boundary and the field values are updated at every time step. Notice that computing the source-side involves computing derivatives at the boundary, and that is done using a high-order, one-sided, finite-difference stencil. (See [4] for more detail.)

This new computational approach allowed us to resolve the apparent disagreement between our results for $\psi_{4}$ and $K$ and the predictions of perturbation theory [6]: we now show agreement in both frequency and magnitude.

Specifically, in the original paper we introduced a fit parameter $W$ that controls the asymptotic deviation of $\psi_{4}$ from a limiting constant magnitude. An asymptotically constant-magnitude $\psi_{4}$ would then be obtained in the limit $W \rightarrow \infty$ as advanced time $v \rightarrow \infty$. With our improved code we find that $W$ would take very high values. (E.g., for $a / M=0.8$ and $m=1$, we find that $W \sim 1,400 M$.) We therefore conclude that we no longer have a reason to introduce $W$, and that in practice $W / M \gg 1$. To illustrate this result, we present in Fig. 7 the asymptotic behavior of $\psi_{4}$ in ingoing Kerr coordinates for a number of $a / M$ and $m$ values.

This behavior affects Figs. 5 and 6 in the original paper. The corrected figures are included. We also correct the values in Table II.

TABLE II. Parameters for the numerically simulated $\psi_{4}$ approaching the Cauchy horizon: for $a / M=0.8$ for various values of $m$ (upper), and for $m=2$ for various values of $a / M$ (lower). The parameter $\omega$ is found from the numerical simulations. The relative difference between $\omega M$ and $m \Omega_{-} M$ is denoted by $\delta$. Data, on the equatorial plane, are presented in ingoing Kerr coordinates.

\begin{tabular}{lccr}
\hline \hline$m$ & $\omega M$ & $m \Omega_{-} M$ & $\delta$ \\
\hline 1 & 1.05 & 1.000 & 0.049 \\
2 & 2.09 & 2.000 & 0.044 \\
3 & 3.14 & 3.000 & 0.046 \\
\hline \hline$a / M$ & $\omega M$ & $m \Omega_{-} M$ & $\delta$ \\
\hline 0.800 & 2.09 & 2.000 & 0.044 \\
0.866 & 1.71 & 1.732 & 0.013 \\
0.917 & 1.48 & 1.528 & 0.032 \\
0.954 & 1.33 & 1.363 & 0.025 \\
0.980 & 1.19 & 1.225 & 0.029 \\
\hline \hline
\end{tabular}


[1] G-S. Jiang and C-W. Shu, J. Comput. Phys. 126, 202 (1996).

[2] S. Gottlieb, C-W. Shu, and E. Tadmor, SIAM Rev. 43, 89 (2001).

[3] S. Gottlieb, D. Ketcheson, and C-W. Shu, Strong Stability Preserving Runge-Kutta and Multistep Time Discretizations (World Scientific, Singapore, 2011).

[4] L. M. Burko and G. Khanna, arXiv:1709.10155.

[5] D. Marolf and A. Ori, Phys. Rev. D 86, 124026 (2012).

[6] A. Ori, Phys. Rev. Lett. 83, 5423 (1999). 